\title{
Colonización nasal bacteriana en población sana de la ciudad de Santiago de Chile: ¿Existe portación de Staphylococcus aureus meticilino resistente comunitario?
}

\author{
Nasal bacterial colonization in healthy people of the city of Santiago of Chile: \\ Is there nasal carriage of community methicillin resistant Staphylococcus aureus?
}

Liesbeth Platzer $\mathbf{M}^{1}$, Carolina Aranís $\mathrm{J}^{2}$, Constanza Beltrán $\mathbf{M}^{2}$, Ximena Fonseca $\mathrm{A}^{2}$, Patricia García $\mathrm{C}^{3}$.

\begin{abstract}
RESUMEN
Introducción: No existe consenso en cuanto a qué se considera flora nasal normal. Recientemente, ha emergido Staphylococcus aureus meticilino resistente comunitario (MRSA-com) en personas sin factores de riesgo conocido, produciendo una alarma sanitaria a nivel mundial.

Objetivo: Determinar la colonización nasal bacteriana y evaluar la presencia de MRSA-com.

Material y método: Estudio prospectivo descriptivo, entre octubre de 2007 y octubre de 2008, en población sana. Se realizó toma de muestra de secreción nasal, incubación e identificación bacteriana por métodos convencionales.

Resultados: Población estudiada con promedio de edad 37,6+15,8 años, 55\% de sexo femenino, y $37,1 \%$ tabaquismo activo. Se obtuvo $73 \%$ de cultivos positivos. Se identificaron 18 especies bacterianas, siendo las más frecuentes Staphylococcus coagulasa negativo (53\%) y Staphylococcus aureus (22,7\%). Se detectó sólo un caso de MRSA, cuyo análisis genético fue negativo para demostrar su origen comunitario.

Discusión: Existe una alta tasa de portación nasal de $S$ coagulasa negativo y $S$ aureus, similar a lo reportado por la literatura internacional. Pese a que la prevalencia encontrada para $S$ aureus es la habitual, no se encontraron muestras positivas a MRSA-com. Lo anterior indica que aún no existe en Chile la diseminación de clones de MRSA-com.
\end{abstract}

Palabras claves: Flora nasal, Staphylococcus aureus meticilino resistente, Staphylococcus aureus meticilino resistente comunitario.

\section{ABSTRACT}

Introduction: Doesn't exists agreement about normal nasal flora. Recently, it has emerged community-associated methicillin-resistant Staphylococcus aureus in people without known risk factors, producing a global health scare.

\footnotetext{
Médico Otorrinolaringólogo. Hospital Regional de Talca.

Médico. Departamento Otorrinolaringología, Pontificia Universidad Católica de Chile.

Médico. Departamento de Laboratorios Clínicos, Pontificia Universidad Católica de Chile.
} 
Aim: Look for normal nasal flora and test the presence of MRSA-com.

Material and method: Descriptive and prospective study, between October 2007 and October 2008, in healthy people. Was taken a sampling from nasal secretion, incubation and bacterial identification by conventional methods.

Results: Studied population, average age $37.6 \pm 15.8$ years, $55 \%$ female and $37.1 \%$ active smoking. We obtained $73 \%$ of positive cultures. We identified 18 bacterial species, the most common was Staphylococcus coagulase-negative (53\%) and Staphylococcus aureus (22.7\%). Was detected only one case of MRSA, and his genetic test was negative to prove community origin.

Discusion: There is a high rate of nasal carriage of Staphylococcus coagulasenegative, like to that reported by international literature. Although the prevalence found for $S$ aureus is the usual, there were no MRSA-com positive samples. This indicates that in Chile there is still no spread of clones of MRSA-com.

Key words: Nasal bacterial colonization, community methicillin resistant Staphylococcus aureus.

\section{INTRODUCCIÓN}

La flora bacteriana de la región anterior de las fosas nasales está compuesta por varias especies que reflejan la flora cutánea (Staphylococcus, Corynebacterium y Difteroides). Sin embargo, no hay consenso en cuanto a qué se considera flora nasal normal, existiendo pocos estudios que la han descrito, siendo éstos disidentes en sus resultados $^{1}$, debido en gran parte a que ésta varía según la exposición ambiental de la persona, por ejemplo, humo de tabaco, o puede cambiar en el tiempo, dependiendo de las características microbiológicas y epidemiológicas de cada país.

El año 2006, Konno y cols ${ }^{2}$ describieron como portación nasal normal a Streptococcus viridans, Staphylococcus aureus (S aureus), Staphylococcus coagulasa negativa y Corynebacterium sp, considerando como punto de corte para asumir su estado como colonización normal su presencia en más del $10 \%$ de los participantes. A su vez, Streptococcus pyogenes, Streptococcus pneumoniae, HaemophiIus influenzae y Moxarella catarrhalis fueron clasificados como patógenos.

Se sabe que $S$ aureus tiene en esta zona su reservorio habitual, desplazando en proporción al resto de los microorganismos y asociándose a Staphylococcus coagulasa negativo, Micrococcus, Corynebacterium y Streptococcus ${ }^{3}$. Según diferentes reportes, entre el $20 \%$ y $30 \%$ de los sujetos sanos pueden estar colonizados de manera persistente 0 transitoria, convirtiéndose en el principal reservorio y fuente de infección para el ser humano.

$S$ aureus también ha sido identificado como un agente intrahospitalario, ya desde la década del $60^{4}$. Actualmente, las cepas de $S$ aureus resistentes a meticilina (MRSA) son un problema de salud pública a nivel mundial, siendo MRSA hospitalario (MRSA-HA) la causa más común de infección nosocomial reportada, transformándose en endemia en la mayoría de los hospitales de EE.UU. y del mundo entero ${ }^{5}$.

Recientemente ha emergido en la comunidad MRSA de origen comunitario en personas sin factores de riesgo conocidos; se denomina MRSAcom para diferenciarlo del hospitalario ${ }^{6}$, y difiere molecularmente dado que posee ${ }^{7}$ :

1. Gen de Panton-Valentine-Leucidina (PVL), que produce una toxina extracelular que destruye leucocitos y causa necrosis tisular ${ }^{8}$, lo que le confiere mayor virulencia,

2. Resistencia heterogénea a meticilina, que determina alguna dificultad en el diagnóstico in vitro,

3. Susceptibilidad a múltiples antibióticos no betalactámicos, y

4. Gran diversidad clonal, dada por el gen mecA de resistencia, en elemento genético móvil denominado Cassette cromosómico estafilocócico mec tipo IV, diferente al que poseen las cepas de MRSA-HA (CCS I-II-III).

Así, estudios revelan que en pacientes con colonización nasal por MRSA-com desarrollan un 
mayor número de infecciones que los portadores de sólo $S$ aureus ${ }^{9}$. La gravedad de la infección por MRSA-com radica en que de no mediar una antibioticoterapia adecuada y rápidamente instituida puede evolucionar a formas graves, sistémicas y en ocasiones mortales en personas inmunocompetentes sin factores de riesgo conocidos.

Lo anterior sumado al número creciente de casos reportados, ha logrado generar una alarma sanitaria a nivel mundial: en Australia el año 2000 se describió una prevalencia de SAMR-com de $10,3 \%$, aumentando hasta $15 \%$ en 2004 . En los Estados Unidos el año 2005, en población infantil, se describió una prevalencia de $9,6 \%{ }^{10}$, y en 2006 de 3\% ${ }^{11}$; en América del Sur se describen distintas prevalencias según el país. Brasil el año 2005 describió en acompañantes de pacientes al servicio de urgencia $(n=600)$ una prevalencia de MRSAcom de $0,7 \%^{12}$. En Argentina, se han descrito casos esporádicos, y en Chile se han reportado a la fecha sólo 7 casos autóctonos ${ }^{13,14}$.

Es por esto, que creemos importante evaluar la presencia de MRSA-com en la comunidad, a modo de vigilancia epidemiológica, no realizada previamente en nuestro país, así como también conocer la flora colonizadora nasal de la población chilena.

\section{OBJETIVOS}

1. Determinar la colonización nasal bacteriana en población sana de Santiago de Chile, y su variación ante la exposición al humo del tabaco.

2. Determinar la existencia de MRSA-com en población sana de Santiago de Chile.

\section{MATERIAL Y MÉTODO}

Se realizó un estudio prospectivo, descriptivo y continuo, entre octubre de 2007 a octubre de 2008. El tamaño muestral requerido fue de 500 pacientes, considerando una prevalencia aproximada del MRSA-com de $5 \%$, según datos obtenidos en distintas series clínicas, para obtener un intervalo de confianza de $\pm 3 \%$, con $95 \%$ de confianza y $80 \%$ de potencia.

\section{Pacientes}

Estudiantes universitarios y acompañantes de pacientes consultantes al Departamento de Otorrinolaringología de la Pontificia Universidad Católica de Chile (PUC). Todos los participantes del estudio firmaron el consentimiento informado, el cual fue aprobado por el comité de ética de la PUC.

Previo a la toma de la muestra, los pacientes debían completar una encuesta epidemiológica donde se preguntaba edad, sexo, actividad laboral, comuna de residencia, antecedentes de tabaquismo, uso de medicamentos intranasales y la presencia de criterios de exclusión, definidos como la presencia de factores de riesgo para MRSA-HA ${ }^{6,15}$, detallados en la Tabla 1.

\section{Toma de muestra}

Se obtuvo una muestra de secreción nasal mediante tórula Stuart estéril que se inserta en la fosa nasal hasta el punto de mayor resistencia correspondiente a los cornetes, aproximadamente 2 a 3 centímetros. Se rota la tórula contra la mucosa nasal en $360^{\circ}$ y se

Tabla 1. Criterios de exclusión al estudio

1. Hospitalización reciente o cirugía (menos de 30 días).

2. Trabajadores en centros de salud.

3. Residencia en asilos o casas de reposo.

4. Diálisis.

5. Uso de catéter.

6. Fiebre $\left(>37,5^{\circ} \mathrm{C}\right)$, rinorrea purulenta, odinofagia.

7. Antecedente de infección respiratoria alta reciente (resfrío, sinusitis, bronquitis, neumonía el último mes).

8. Uso reciente de: antibióticos los últimos 2 meses, corticoides orales o nasales, inmunosupresores, quimio o radioterapia. 
repite el proceso en fosa nasal contralateral. La muestra se lleva en medio de transporte hasta el laboratorio, en menos de 24 horas, donde es sembrada en tres medios de cultivos diferentes: agar sangre, agar chocolate y agar Mac Conkey. Las placas de medios de cultivos fueron incubadas a $35^{\circ} \mathrm{C}$ por 16 24 horas. La identificación bacteriana se realizó según los procedimientos definidos en el laboratorio de microbiología y la susceptibilidad a cloxacilina, se realizó por el método de dilución en agar (método de referencia), utilizando oxacilina. La Figura 1 esquematiza el proceso de toma de muestras.

En caso de identificarse MRSA, la muestra debía ser congelada para realizar su posterior análisis genético en el Instituto de Salud Pública de Chile, para la confirmación del origen comunitario. Las concentraciones inhibitorias mínimas ensayadas fueron las recomendadas por el CLSI (Tabla 2).

\section{Análisis estadístico}

Se utilizó el Programa SPSS 13.0, considerando diferencia estadísticamente significativa valor $p<0,05$. Se utilizó Chi para variables categóricas y ANOVA cuando existieron más de 2 variables en el análisis.

\section{RESULTADOS}

Se estudió a 500 individuos, de los cuales 454 $(91,8 \%)$ cumplían los criterios de inclusión, por lo que se procedió a tomarles muestra de secreción nasal. Todos firmaron el consentimiento informado.

El promedio de edad de la población estudiada fue $37,6 \pm 15,8$ años, con un rango etario de 13 a 78 años, siendo $55 \%$ de sexo femenino.

El $37,1 \%(n=161 / 434)$ de los participantes tenía antecedentes de tabaquismo activo.

No se encontraron diferencias significativas en cuanto a especies bacterianas cultivadas, al comparar por edad, profesión y comuna de residencia.

Durante el estudio no se presentaron efectos adversos ni complicaciones derivados de la toma de muestra de secreción nasal.

\section{a) Colonización nasal bacteriana}

El 73\% (331/454) de los cultivos realizados fueron positivos. Se identificaron 18 especies bacterianas diferentes, las cuales se muestran en la Tabla 3. La bacteria más frecuentemente aislada fue Staphylococcus coagulasa negativo en $53 \%$, seguido de $S$ aureus en $22,7 \%$.

En el $65 \%$ de los cultivos positivos se identificó solo una especie bacteriana, en $33 \%$ de los cultivos se identificó a 2 especies bacterianas y en sólo el $2,8 \%$ de las muestras se identificaron 30 más especies bacterianas.

Se encontraron algunas asociaciones estadísticamente significativas:

1. Del total de cultivos positivos para Staphylococcus coagulasa negativo, sólo el 13,8\% (33/240) presentó colonización concomitante de $S$ aureus ( $p=0,000)$, versus $32,7 \%(70 / 214)$ en cultivos negativos para Staphylococcus coagulasa negativo.

2. Del total de cultivos positivos para Difteroides sp, el $95 \%$ de éstos se asocia a la presencia de Staphylococcus coagulasa negativo y sólo el $10 \%$ de las muestras positivas a Difteroides $\mathrm{sp}$ se asocia a $S$ aureus $(p<0,001)$.

3. La población no fumadora presenta $55,7 \%$ (152/ 273) de cultivos positivos para Staphylococcus coagulasa negativo, versus $45,3 \%$ (73/161) en la población fumadora, siendo esta diferencia estadísticamente significativa ( $p=0,047$ ).

No se encontró asociación estadísticamente significativa entre otras especies bacterianas. No hubo diferencias significativas de colonización nasal bacteriana, al comparar por edad, profesión y comuna de residencia.

\section{b) Colonización por MRSA}

Se detectó sólo un caso de MRSA $(0,24 \%)$ que fue resistente a oxacilina (R4), a gentamicina (R16) y a ciprofloxacino (R4). La muestra pertenece a un hombre de 60 años, sano, sin factores de riesgo conocidos para MRSA, sin antecedente de viajes ni contacto con extranjeros. El análisis genético fue negativo para demostrar el origen comunitario.

\section{DISCUSIÓN}

\section{a) Colonización nasal bacteriana}

Dentro de las limitaciones del estudio se debe considerar que es una muestra poco representativa 


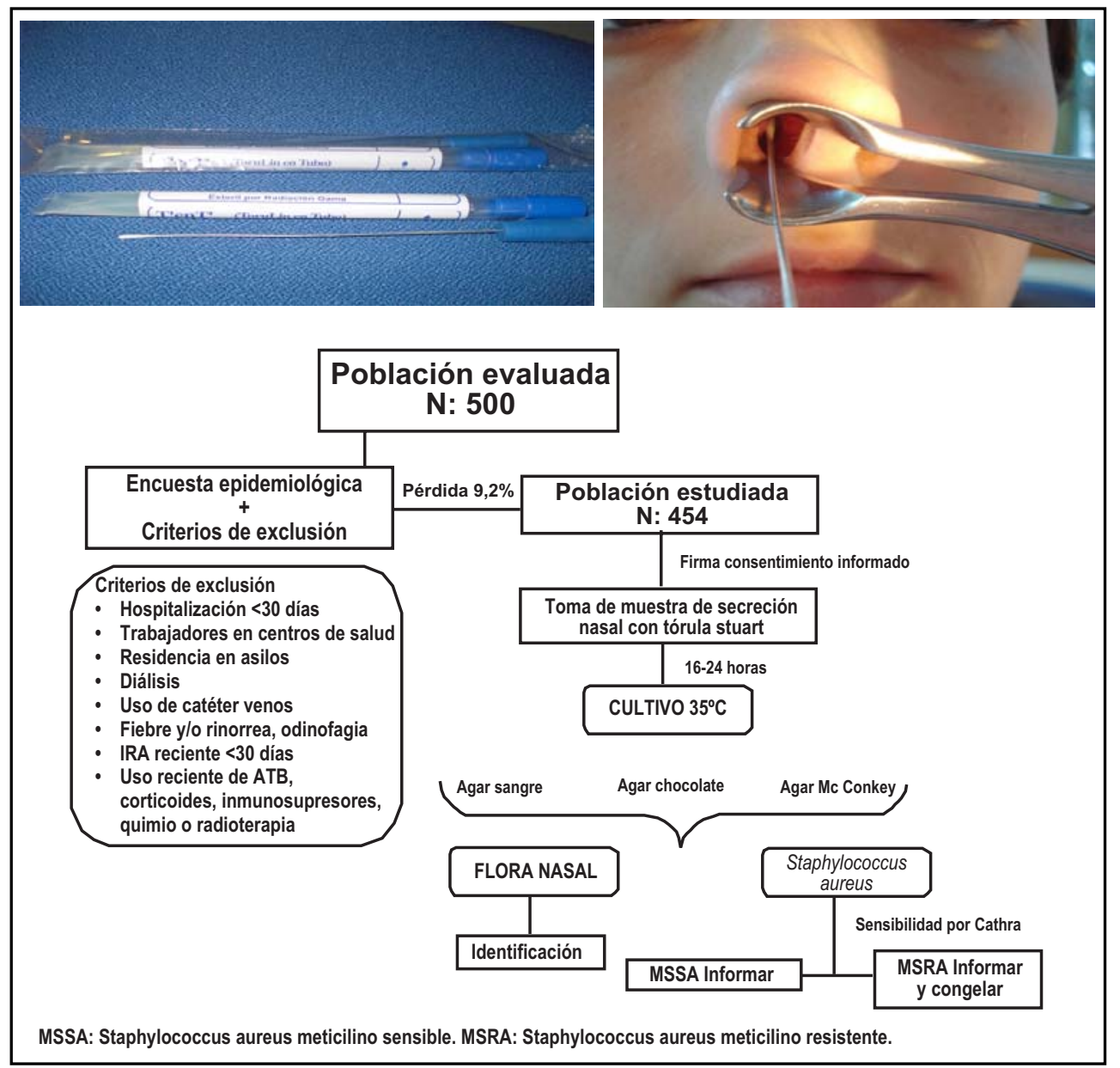

Figura 1. Proceso de tomas de muestras y posterior análisis en laboratorio.

Tabla 2. Concentraciones inhibitorias mínimas ensayadas recomendadas por el CLSI

\begin{tabular}{|lcc|}
\hline Microorganismo & \multicolumn{2}{c|}{$\begin{array}{c}\text { Cloxacilina (oxacilina) } \\
\text { Concentración inhibitoria mínina (ug/ml) } \\
\text { Resistente }\end{array}$} \\
\hline Staphyloceptible & $\leq 2$ & $\geq 4$ \\
Staphylococus aureus & $\leq 0,25$ & $\geq 0,5$ \\
\hline
\end{tabular}

a nivel nacional, realizado sólo en la región metropolitana y en centros de la red de salud de la PUC, Io cual en cuanto a calidad metodológica está muy por debajo de un estudio multicéntrico, el cual abarcaría una mayor y diversa población. Sin embargo, sirve a futuro como referente.
En relación a la colonización nasal, nuestro trabajo encontró 18 especies bacterianas diferentes, siendo la más frecuente en forma aislada 0 asociada a otras bacterias Staphylococcus coagulasa negativo. Le sigue en orden de frecuencia $S$ aureus, Difteroides sp, Corynebacterium sp y 
Tabla 3. Bacterias aisladas en secreción nasal

\begin{tabular}{|lrr|}
\hline Bacterias aisladas en secreción nasal & Total $\mathrm{n}$ : 454 & $\%$ \\
\hline Cultivos negativos & 123 & 27,2 \\
Staphylococcus coagulasa (-) & 240 & 53,0 \\
Staphylococcus aureus & & \\
(meticilino sensible) & 103 & 22,7 \\
Corynebacterium sp & 25 & 5,5 \\
Difteroides sp & 33 & 7,3 \\
Streptococcus grupo viridans & 20 & 4,4 \\
Neisseria sp & 6 & 1,4 \\
Streptococcus pneumoniae & 4 & 0,9 \\
E coli & 3 & 0,7 \\
Haemophilus sp & 3 & 0,7 \\
Haemophilus influenzae & 2 & 0,4 \\
Bacillus sp & 1 & 0,2 \\
Streptococcus Grupo G & 1 & 0,2 \\
SAMR & 1 & 0,2 \\
Enterobacter aerogenes & 1 & 0,2 \\
Haemophilus parahaemolyticus & 1 & 0,2 \\
Klebsiella pneumoniae & 1 & 0,2 \\
Klebsiella oxytoca & 1 & 0,2 \\
Proteus sp & 1 & 0,2 \\
Moraxella sp & 1 & 0,2 \\
\hline
\end{tabular}

Streptococcus grupo viridans, lo que es similar a lo descrito por otros autores. También se observó la presencia de población asintomática colonizada por bacterias consideradas patógenas ${ }^{2}$, como $E$ coli, Streptococcus pneumoniae y Haemophilus influenzae, lo cual podría explicarse por una posible portación transitoria, correspondiendo así sólo a portadores asintomáticos, o por la toma del cultivo en el periodo de incubación asintomático de la enfermedad.

Sin embargo, la relación entre colonización e infección no ha sido completamente entendida, pero está asociada a factores intrínsecos del hospedero como también a factores propios de la bacteria. Es discutible que $S$ aureus sea considerado como flora normal ya que algunos autores ${ }^{16}$ han estudiado la correlación entre la portación nasal de este microorganismo y la presencia de bacteriemia por $S$ aureus, encontrándose clonalidad entre ambas cepas (nasal y la de sangre) en $80 \%$, por 10 cual la portación nasal de $S$ aureus podría considerarse como un factor asociado a bacteriemia.

No hay diferencias significativas en la flora nasal, al comparar por edad, profesión y comuna de residencia. Sí se encontró diferencias significativas al evaluar el antecedente de tabaquismo, ya que la población fumadora presenta menor número de cepas bacterianas (Staphylococcus coagulasa negativo) en relación a la población no fumadora de las mismas características ( $p<0,04$ ), lo que concuerda con lo descrito en la literatura. Un estudio publicado por Brook ${ }^{17}$ en 2007 muestra que en los cultivos nasofaríngeos de fumadores existe un alto número de patógenos y una baja cantidad de flora normal, lo que se relaciona a un mayor riesgo de presentar infecciones respiratorias y colonización de la cavidad oral con microorganismos patógenos, siendo esta situación reversible al suspender el tabaco totalmente.

Diversos estudios describen una baja incidencia de colonización por $S$ aureus en pacientes sanos colonizados por Corynebacterium sp y Staphylococcus coagulasa negativo. Esta interacción ha sido demostrada en experimentos in vivo y podría sugerir que ambas bacterias antagonizan la colonización nasal por $S$ aureus ${ }^{18}$. Nuestro estudio muestra que la portación nasal de $S$ aureus se correlaciona negativamente y en forma significativa $(p<0,001)$ con la presencia de colonización nasal por Difteroides sp y Staphylococcus epidermidis, lo que apoya la hipótesis de un antagonismo en el nicho biológico de ambas especies.

Por último, cabe destacar que varios reportes internacionales han mencionado la posibilidad de que la colonización nasal por $S$ aureus influiría a través de superantígenos en los síntomas de la rinitis alérgica perenne, por lo que sería interesante estudiar en forma prospectiva qué pasa en los pacientes portadores de $S$ aureus nasal, su relación con síntomas de rinitis alérgica perenne 0 la falta de respuesta a tratamiento a ella ${ }^{19}$.

\section{b) Colonización por MRSA}

Nuestros resultados coinciden con la literatura internacional, en relación a la alta tasa de portación nasal de Staphylococcus aureus multisensible $(22,7 \%)$. Según diferentes estudios, la colonización nasal por Staphylococcus aureus alcanza al $20 \%-30 \%$ de la población general y no implica la presencia de infección verdadera ${ }^{20}$.

Pese a que la prevalencia encontrada para $S$ aureus es la habitual, hubo sólo un caso probable 
de MRSA-com $(0,24 \%)$, en el cual finalmente no se detectó el gen de PVL, descartándose así su origen comunitario.

Es por esto que creemos que MRSA-com, no es aún un problema de salud pública en nuestro país, y no existe información suficiente que justifique la realización rutinaria de cultivos nasales en la comunidad en búsqueda de MRSA-com.

No obstante lo anterior, se han reportado un número creciente de casos y en diferentes poblaciones, lo que hace pensar que pronto aparecerán cepas de MRSA-com, por lo que debemos mantenernos en constante vigilancia epidemiológica.

\section{CONCLUSIONES}

Nuestros resultados coinciden con la literatura internacional, en relación a la flora nasal encontrada y a la alta tasa de portación nasal de $S$ aureus.

No hay diferencias significativas en la flora nasal, al comparar por edad, profesión (excluyendo trabajadores de salud) y comuna de residencia.

La población fumadora presenta menor número de cepas bacterianas en relación a la población no fumadora de las mismas características, lo que concuerda con lo descrito en la literatura $(p<0,04)$.

La portación nasal de $S$ aureus se correlaciona negativamente con la frecuencia de colonización por Difteroides sp y $S$ coagulasa negativo, lo que sugiere que ambas bacterias antagonizan la colonización por $S$ aureus $(p<0,001)$.

No encontramos MRSA-com en población sana de Santiago de Chile, por lo que podemos afirmar que esta cepa como causante de infecciones, no es aún un problema de salud pública en nuestro país. Así, no se justificaría estudiar la colonización nasal en todas las personas infectadas 0 de la comunidad, ni menos la utilización rutinaria de tratamientos de descolonización nasal.

\section{Agradecimientos}

El presente trabajo pudo realizarse gracias al financiamiento entregado por el Concurso de Residentes del Centro de Investigaciones Médicas (CIM) de la PUC, el Concurso de Laboratorios Clínicos e Infectología PUC y el Concurso de Investigación de la Sociedad Chilena de Otorrinola- ringología (SOCHIORL). Agradecemos del mismo modo al personal paramédico y técnicos de los Departamentos de Otorrinolaringología y de Laboratorios Clínicos por su aporte a la realización del estudio.

\section{BIBLIOGRAFÍA}

1. Rasmussen Tt, Kirkeby LP, Poulsen K, Reinholdt J, KILIAN M. Resident aerobic microbiota of the adult human nasal cavity. APMIS 2000; 108 (10): 663-75.

2. Konno M Y cols. Study of upper respiratory tract bacterial flora: first report. Variations in upper respiratory tract bacterial flora in patients with acute upper respiratory tract infection and healthy subjects and variations by subject age. $J$ Infect Chemother 2006; 12 (2): 83-96.

3. Usviatsov BIA, Parshuta LI, Bukharin OV. Microbial biocenosis of the nasal mucosa in healthy persons and in Staphylococcus carriers. Zh Mikrobiol Epidemiol Immunobiol 2000; (5): 65-9.

4. JORGENSEN JH. Laboratory and epidemiologic experience with methicillin-resistant Staphylococcus aureus in the USA. Eur J Clin Microbiol 1986; 5: 693-6.

5. Chambers HF. The changing epidemiology of Staphylococcus aureus? Emerg Infect Dis 2001; 7: 178-82.

6. Beaglehole $R$, Bonita $R$, Kuellstrom $T$. Epidemiología básica. Washington: OPS; 1994.

7. BABA Y COLS. Genome and virulence determinants of high virulence community-acquired MRSA. Lancet 2002; 359: 1819-27.

8. Nimmo GR Y coLs. Methicillin-resistant Staphylococcus aureus in the Australian community: an evolving epidemic. Med J Aust 2006; 184(8): 384-8.

9. Yl Lee, T Cesario, A Pax, C Tran, A Ghouri, LD THRUPP. Nasal colonization by Staphylococcus aureus in active, independent, community seniors. Age and Ageing 1999; 28: 229-32.

10. Creech CB $2^{\text {no }}$, Kernodle DS, Alsentzer A, Wilson $C$, EDWARDS KM. Increasing rates of nasal carriage of methicillin-resistant Staphylococcus aureus in healthy children. Pediatr Infect Dis $J$ 2005; 24: 617-21. 
11. Creech CB 2no, Talbot tR, Schaffner W. Community-Associated Methicillin-Resistant Staphylococcus aureus: The Way to the Wound Is through the Nose. J Infect Dis 2006; 193 (2): 169-71.

12. Ribeiro J, Boyce JM, Zancanaro PQ. Prevalence of methicillin-resistant Staphylococcus aureus (MRSA) among patients visiting the emergency room at a tertiary hospital in Brazil. Braz $J$ Infect Dis 2005; 9 (1): 52-5.

13. Aylwin M, Serri M, García P, Sader H, Pérez C. Staphylococcus aureus meticilino resistente asociado a la comunidad en Chile. Libro de Resúmenes XXIV Congreso Chileno de Infectología, 2007; Abstr C021, pág 42.

14. Noriega LM, González P, Hormazábal JC, Pinto C, Canals M, Munita JM, Thompson L, Marcotti A, Pérez J, Ibáñez D, Araya P, Canals C, Vial P. Staphylococcus aureus comunitario resistente a cloxacilina: Comunicación de los primeros cinco casos descritos en Chile. Rev Méd Chile 2008; 136: 885-91.
15. NAIMI TS Y cols. Comparison of community- and health care-associated methicillin-resistant Staphylococcus aureus infection. JAMA 2003; 290 (22): 2976-84.

16. Von eiff C, Becker K, Machika K et al. Nasal carriage as a source of Staphylococcus aureus bacteremia. Study group. N Englan J Med 2001; 344: 11-6.

17. Brook, Itzhak MD, Gober, Alan E. MD. Effect of Smoking Cessation on the Microbial Flora. Arch Otolaryngol Head Neck Surg 2007; 133: 135-8.

18. Lina G, Boutite F, Tristan A, et al. Bacterial competition for human cavity colonization: rol of Staphylococcal agr Alleles. Applied and Environmental Microbiology 2003; (69): 18-23.

19. Uehara Y, Nakama $H$, Agematsu K et al. Bacterial interferente among nasal inhabitants: erradication of Staphylococcus aureus from nasal cavities by artificial implantation of Corynebacterium sp. $J$ Hosp Infect 2000; 44 (2): 127-33.

20. ARCHER GL. Staphylococcus aureus: a wellarmed pathogen. Clin Infect Dis 1998; 26: 1179-81.

Dirección: Carolina Aranís Jiménez

Av. Suecia 750, Depto. 508. Providencia, Santiago

E mail: caaranis@gmail.com 